\title{
基于自适应遗传算法的LED器件多应力条件下 寿命快速评估系统模型
}

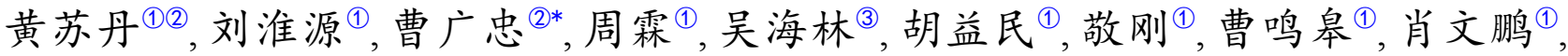
刘岩 (1)*

(1) 深圳清华大学研究院广东省光机电一体化重点实验室, 深圳清华大学研究院深圳市光机电一体化重点实验室, 深圳 518060;

(2) 深圳大学深圳电磁控制重点实验室, 深圳 518060;

(3) 深圳大学材料学院, 深圳 518060;

*E-mail: gzcao@szu.edu.cn; liuy@tsinghua-sz.org

收稿日期: 2016-02-07; 接受日期: 2016-06-16; 网络出版日期: 2016-09-01

国家重点基础研究发展计划(编号: 2011CB013106)、国家自然科学基金(批准号: 51275312)、国家科技支撑计划(编号: 2014BAH23F04)、 深圳市科技计划(编号: JSGG20141015153303491, KC2014JSJS025A)资助项目

摘要发光二极管(light-emitting diode, LED)作为正在兴起的固态冷光源具有效率高、寿命长、体积小、响 应快、抗振、驱动电压低、绿色环保等特点, 在照明光源、仪表指示等领域显示出较大的发展潜力.针对LED 器件寿命在实际使用环境中应力影响因素复杂、加速寿命试验模型考虑的应力因素不全面以及LED器件寿命 预测模型准确性低等问题, 本文提出了一种LED器件多应力条件下寿命快速评估系统模型. 该模型适用于各种 工艺制造的不同结构的LED器件. 以光效衰减 $30 \%$ 为寿命依据, 以环境温度、湿度和驱动电流为输入量、LED 器件寿命为输出量, 采用设计的自适应遗传算法确定被测器件的辨识参数.利用构建的加速寿命试验获得较高 温度湿度电流应力下的少量样本数据, 基于得到的样本数据辨识该模型的未知参数, 获得了模型. 在常温常湿 常电流应力下, 通过该模型进行了 LED 器件寿命预测. 研究结果表明: 在任意温度湿度电流应力下, 提出的模型 可以快速较精确地实现LED器件寿命预测; 在常温常湿常电流应力下, 基于提出的模型得到的LED器件寿命接 近于LED行业平均技术水平的LED预期寿命; 提出的模型应用于LED器件寿命可靠性测评具有实用性, 并可继 续拓展成为纳入更多应力的模型.

关键词 发光二极管, 寿命快速评估, 加速寿命试验, 自适应遗传算法, 参数辨识

\section{1 引言}

发光二极管(light-emitting diode, LED)是一种新型 固态冷光源, 与传统的白炽灯、苂光灯等照明光源相 比, 具有效率高、寿命长、体积小、响应快、抗振、
驱动电压低、绿色环保等优点 ${ }^{[1 \sim 5]}$. 普通照明光源是 LED最具发展前景的一种应用, 目前LED光源已经实 用化并产业化, 取代传统照明光源已成为不可逆转的 趋势 ${ }^{[6 \sim 10]}$. LED器件能否被真正广泛应用于照明光源, 其关键因素是可靠性问题的解决 ${ }^{[10 \sim 13]}$. LED的可靠性

引用格式: 黄苏丹, 刘淮源, 曹广忠, 等. 基于自适应遗传算法的LED器件多应力条件下寿命快速评估系统模型. 中国科学: 技术科学, 2016, 46: 940-949 Huang S D, Liu H Y, Cao G Z, et al. Modeling of the rapid lifetime assessment system with multiple stresses for LED devices using adaptive genetic algorithm (in Chinese). Sci Sin Tech, 2016, 46: 940-949, doi: 10.1360/N092016-00058 
包括光性能、电性能、热性能、寿命等, 寿命是其最 常用且代表性的可靠性参数.

LED器件的理论寿命可达 10 万小时, 但实际使用 寿命一般为 $2 \sim 3$ 万小时甚至几千小时, 远未达到理论寿 命 ${ }^{[14-16]}$. 由于LED器件的寿命较长, 采用直接施加额定 电流直到寿命终止的长期通电老化的传统测试方式, 成本高、效率低且不符合实际工业应用. 因此, 半导体 照明行业普遍采用对LED器件施加高应力, 通过加速 寿命试验方法测量LED器件在高应力下的寿命, 再应 用外推法计算正常应力下的寿命. 进行加速寿命试验 之前, 需要建立加速应力模型. 加速应力模型主要有幂 次定律模型, Arrhenius模型, Eyring模型, Peck模型等 ${ }^{[17]}$. 以温度为单一应力环境因素构建的Arrhenius模型是迄 今被广泛使用的加速应力模型 ${ }^{[1,19]}$. LED器件在实际工 作过程中, 除了受温度这一主要环境因素作用外, 还受 到湿度、温度冲击、振动等环境因素和驱动电流的作 用. 因此, 反映温度、湿度和驱动电流等多应力因素的 加速应力模型更符合实际. 加速寿命试验方法的LED 标准包括国际电工委员会(International Electrotechnical Commission, IEC)标准、国际照明委员会(International Commission on Illumination, CIE)标准、北美照明工程 协会(Illuminating Engineering Society of North America, IESNA)的LM- $80^{[20]}$ 和TM-2 $1^{[21]}$ 标准等. 对于 LED器件 流明维持寿命的评估, IESNA 的LM-80和TM-21标准 评测时间要求达到6000 10000 h.

IESNA的LM-80 和TM-21中关于LED寿命预测的 标准为代表的单应力环境因素模型与实际使用情况 的多应力环境因素不符. 针对该问题, 以Arrhenius单 应力环境因素模型为基础, 纳入环境湿度、驱动电流 应力因素, 利用Peck模型, 提出了一种LED器件多应力 条件下寿命快速评估系统模型. 该模型以环境温度、 湿度和驱动电流为输入、LED寿命为输出, 表示为一 个具有 5 个未知参数的非线性方程, 可以预测任意温 度、湿度和电流应力下的LED寿命, 特别是常温常湿 常电流应力下的LED寿命. 利用较高温度、湿度和电 流应力下的样本数据, 对该模型的 5 个未知参数进行 系统参数辨识, 从而建立LED器件多应力条件下寿命 快速评估系统模型. 由于非线性的LED器件多应力条 件下寿命快速评估系统建模应适应加速寿命试验的 测量样本, 而测量样本一般测量时间长且样本少. 所 以, 该建模应满足基于极少样本数据的强非线性建模,
需要寻求一种具有少样本、全局寻优、强非线性建 模特性的系统参数辨识方法. 自适应遗传算法系统参 数辨识法需要的信息量少, 具有全局寻优、并行性、 速度快和不需要目标函数可微或连续等优点 ${ }^{[2226]}$. 因 此, 采用自适应遗传算法辨识LED器件多应力条件下 寿命快速评估系统模型的未知参数.

由于产业化的LED器件的材料、工艺、结构等 千差万别, 一个评估模型很难应用于所有的LED器件. 实际应用中, 应对每种LED器件产品辨识其特定的模 型参数, 构建每种LED器件多应力条件下寿命快速评 估系统模型, 并对每种LED器件进行寿命预测. 因此, 需要能够快速进行温度、湿度、电流、光参数等测 量的设备与评估模型配套, 使企业能够高效地对每种 新产品作出测评.

\section{LED器件多应力条件下寿命快速评估系 统模型}

电子器件一般采用的加速寿命试验假设试验中 只存在单一加速应力, 被广泛使用的以环境温度为单 一加速应力的Arrhenius模型为

$$
\tau=\tau_{\mathrm{r}} \mathrm{e}^{\frac{E_{\mathrm{a}}}{K T}},
$$

式中, $\tau$ 为服役时间, $\tau_{\mathrm{r}}$ 为时间常数, $E_{\mathrm{a}}$ 为激活能, $K=1.3806488 \times 10^{-23} \mathrm{~J} / \mathrm{K}$ 为玻尔兹曼常数, $T$ 为绝对温度.

Arrhenius模型的温度加速系数 $A_{\mathrm{T}}$ 为

$$
A_{\mathrm{T}}=\frac{\tau_{\mathrm{T} 2}}{\tau_{\mathrm{T} 1}}=\mathrm{e}^{\frac{E_{\mathrm{a}}}{K}}\left(\frac{1}{T_{2}}-\frac{1}{T_{1}}\right),
$$

式中, $\tau_{\mathrm{T} 1}, \tau_{\mathrm{T} 2}$ 分别为温度 $T_{1}$ 和 $T_{2}$ 应力下的LED器件寿命.

若加速寿命试验存在环境温度和湿度 2 个加速应 力, 则使用Peck模型. 以温度和湿度为加速应力的Peck 模型为

$$
\tau=\tau_{0} \mathrm{e}^{\frac{E_{\mathrm{a}}}{K T}} H^{-m},
$$

式中, $H$ 为相对湿度百分率, $m$ 为常系数, $\tau_{0}$ 为材料常数.

Peck模型的加速系数 $A_{\mathrm{TH}}$ 为

$$
A_{\mathrm{TH}}=\frac{\tau_{\mathrm{TH} 2}}{\tau_{\mathrm{TH} 1}}=\mathrm{e}^{\frac{E_{\mathrm{a}}}{K}\left(\frac{1}{T_{2}}-\frac{1}{T_{1}}\right)}\left(\frac{H_{1}}{H_{2}}\right)^{m},
$$

式中, $\tau_{\mathrm{TH} 1}$ 和 $\tau_{\mathrm{TH} 2}$ 分别为温度 $T_{1}$, 湿度 $H_{1}$ 和温度 $T_{2}$, 湿度 $\mathrm{H}_{2}$ 应力下的LED器件寿命. 
参考Arrhenius模型和Peck模型, 构建考虑环境温 度、湿度和驱动电流加速应力的模型为

$$
\tau=\tau_{0} \mathrm{e}^{\frac{E_{\mathrm{a}}}{K T}} I^{-n} H^{-m},
$$

式中, $I$ 为驱动电流, $n$ 为常系数.

以Arrhenius单应力环境因素模型为基础, 纳入环 境湿度和驱动电流应力因素, 利用Peck模型和式(5), 构 造LED器件寿命多应力因素模型. 构建以环境温度 $T$, 湿度 $H$ 和驱动电流 $I$ 为已知输入量, 预测寿命 $\tau$ 为输出量 的LED器件多应力条件下寿命快速评估系统模型为

$$
\begin{aligned}
\tau(T, H, I)= & \tau_{\mathrm{r}} \mathrm{e}^{\frac{E_{\mathrm{a}}}{K T}} I^{-n} H^{-m}+A \tau_{\mathrm{r}} \mathrm{e}^{\frac{2 E_{\mathrm{a}}}{K T}} I^{-2 n} \\
& +B \tau_{\mathrm{r}} \mathrm{e}^{\frac{2 E_{a}}{K T}} H^{-2 m},
\end{aligned}
$$

式中, $T \in[273.15,398.15] \mathrm{K}, H \in[0,100] \% \mathrm{RH}, I \in[0,0.7]$ $\mathrm{A}, \tau_{\mathrm{r}} \in\left[10^{-5}, 10^{-3}\right] \mathrm{h} \mathrm{A}^{\mathrm{n}}$ 是一个与器件有关的未知参数, $A \in\left[-2 \times 10^{-6}, 2 \times 10^{-6}\right] \mathrm{A}^{\mathrm{n}}, B \in\left[-2 \times 10^{-6}, 2 \times 10^{-6}\right] \mathrm{A}^{\mathrm{n}}, m \in[0,2]$ 和 $n \in[0,2]$ 为模型的未知参数.

$\tau_{\mathrm{r}}, A, B, m$ 和 $n$ 为 $\mathrm{LED}$ 器件多应力条件下寿命快速 评估系统模型的 5 个未知参数, 须对其进行参数辨识. 基于式(6), LED器件多应力条件下寿命快速评估系统 模型的结构, 采用自适应遗传算法, 辨识模型参数 $\tau_{\mathrm{r}}$, $A, B, m$ 和 $n$, 从而建立 $\mathrm{LED}$ 器件多应力条件下寿命快速 评估系统模型 $\tau(T, H, I)$, 实现任意温度 $T$, 湿度 $H$, 电流 $I$ 应力下的LED寿命预测, 特别是常温常湿常电流下的 LED寿命预测.

\section{3 基于自适应遗传算法的 LED器件多应力 条件下寿命快速评估系统建模}

遗传算法的基本思想来源于达尔文的进化论和 门德尔的遗传学说, 遵循“物竞天择, 适者生存”原则. 为了提高算法的执行效率并保持每代种群的多样性 与差异性, 将自适应策略引入传统遗传算法, 从而在 保证种群多样性和健壮性的同时, 提高算法效率. 由 于实数编码无需数据类型转换、精度高、物理意义 明确, 因而采用实数编码对染色体进行编码. 利用实 数编码的染色体, 不需要解码, 直接可表示成个体. 因 此, 应用于LED器件多应力条件下寿命快速评估系统 模型的自适应遗传算法, 染色体和个体均表示为 $\left\{\tau_{\mathrm{r}}, A\right.$, $B, m, n\}$. 较大的种群大小和迭代次数可提高全局寻优
性, 但过大的种群大小和迭代次数使泛化性能降低且 计算耗时. 因此, 需结合实际算法, 兼顾全局寻优性、 泛化性能和计算快速性, 通过仿真研究确定种群大小 和迭代次数. 基于自适应遗传算法的LED器件多应力 条件下寿命快速评估系统建模流程如图1所示.

\section{1 适应度函数}

适应度函数用于评价个体的优劣程度. 一般适应 度(适应度函数值)越大表明个体越好. 根据适应度大 小对个体进行选择, 以保证适应性更好的个体有更多 的机会繁殖后代,使优良特性得以遗传.

LED器件多应力条件下寿命快速评估系统模型的 预测寿命与LED真实寿命之间的误差越小, 则构建的 LED器件多应力条件下寿命快速评估系统模型越准 确, 因此, 选择适应度函数为

$$
f\left(\tau_{j}^{*}\right)=-\sum_{j=1}^{n}\left|\tau_{j}-\tau_{j}^{*}\right|,
$$

式中, $n$ 为样本个数, $\tau_{j}$ 为第 $j$ 个样本的LED真实寿命, $\tau_{j}^{*}$ 为第 $j$ 个样本的LED预测寿命.

式(7)表明, 适应度较大的个体优于适应度较小的 个体.

\section{2 选择操作}

选择操作是对种群个体进行优胜劣汰的选择, 适 应度较大的个体被遗传到下一代种群的概率较大, 适 应度较低的个体被遗传到下一代种群的概率较小. 采 用排序选择结合轮盘奢选择的方法, 对种群进行选择 操作, 相应的算法流程如图2所示.

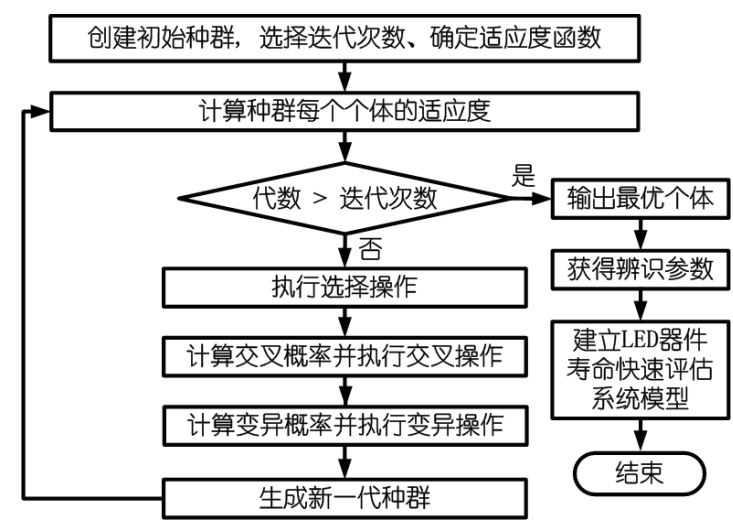

图 1 基于自适应遗传算法的LED器件多应力条件下寿 命快速评估系统建模流程 


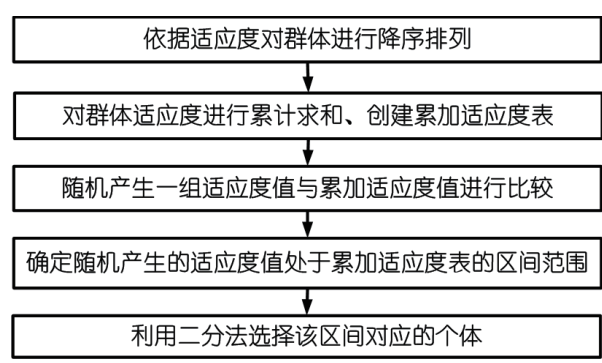

图 2 选择操作流程

\section{3 交叉操作}

交叉操作是指两个相互配对的染色体按某种方 式相互交换部分基因, 从而形成两个新的个体. 交叉 操作是产生新个体的主要方法, 决定了遗传算法的全 局搜索能力.

采用非一致交叉生成新的个体, 该交叉操作表示 为

$$
\left\{\begin{array}{l}
x_{1}^{*}=a_{2} x_{1}+\left(1-a_{2}\right) x_{2}, \\
x_{2}^{*}=a_{2} x_{2}+\left(1-a_{2}\right) x_{1},
\end{array} a_{1}<p_{c}\right.
$$

式中, $x_{1}$ 和 $x_{2}$ 为进行交叉操作的两个个体, $x_{1}{ }^{*}$ 和 $x_{2}{ }^{*}$ 为交 叉操作产生的两个新个体, $a_{1} \in[0,1]$ 和 $a_{2} \in[0,1]$ 为随机 数, $p_{\mathrm{c}}$ 为交叉概率.

利用图3的自适应交叉概率进行交叉操作, 该交 叉概率表示为 ${ }^{[24]}$

$$
P_{\mathrm{c}}=\left\{\begin{array}{l}
P_{\mathrm{c} 1}, f_{\max }<f_{\mathrm{avg}} ; \\
P_{\mathrm{c} 1}-\left(P_{\mathrm{c} 1}-P_{\mathrm{c} 2}\right) \frac{\left(f-f_{\mathrm{avg}}\right)}{\left(f_{\max }-f_{\mathrm{avg}}\right)}, f_{\max } \geq f_{\mathrm{avg}},
\end{array}\right.
$$

式中, $P_{\mathrm{c} 1}=0.99$ 为交叉概率上限, $P_{\mathrm{c} 2}=0.49$ 为交叉概率 下限, $f$ 为进行交叉操作两个染色体适应度的较大值, $f_{\text {avg }}$ 为当代种群的平均适应度, $f_{\text {max }}$ 为当代种群的最大

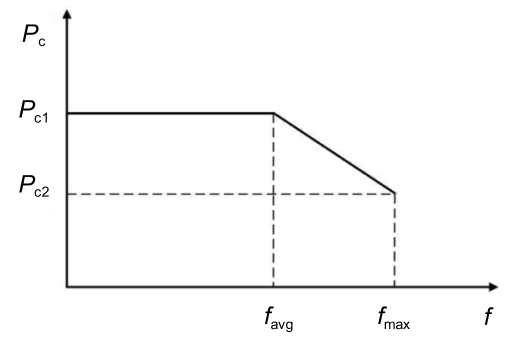

图 3 自适应交叉概率
适应度。

\section{4 变异操作}

变异操作是产生新个体的辅助方法, 决定了遗传 算法的局部搜索能力.

采用的变异操作表示为

$$
\left\{\begin{array}{l}
x^{*}=x+a_{4}\left(x_{\min }-x\right), a_{4} \geq 0.5, a_{3}<p_{\mathrm{m}} \\
x^{*}=x+a_{4}\left(x_{\max }-x\right), a_{4}<0.5, a_{3}<p_{\mathrm{m}},
\end{array}\right.
$$

式中, $x$ 为进行变异操作的个体, $x^{*}$ 为变异操作产生的 新个体, $a_{3} \in[0,1]$ 和 $a_{4} \in[0,1]$ 为随机数, $x_{\min }$ 和 $x_{\max }$ 分别为 个体实数编码的下限和上限, $p_{\mathrm{m}}$ 为变异概率.

通过图4的自适应变异概率进行变异操作, 该变 异概率表示为 ${ }^{[24]}$

$$
P_{\mathrm{m}}=\left\{\begin{array}{l}
P_{\mathrm{m} 1}, f^{\prime}<f_{\text {max }} ; \\
P_{\mathrm{m} 1}-\left(P_{\mathrm{m} 1}-P_{\mathrm{m} 2}\right) \frac{\left(f_{\text {max }}-f^{\prime}\right)}{\left(f_{\text {max }}-f_{\text {avg }}\right)}, f^{\prime} \geq f_{\max },
\end{array}\right.
$$

式中, $P_{\mathrm{m} 1}=0.1$ 为变异概率的上限, $P_{\mathrm{m} 2}=0.001$ 为变异概 率的下限, $f^{\prime}$ 为进行交叉操作染色体的适应度.

\section{4 模型验证}

\section{1 自适应遗传算法的仿真验证}

根据式(6)的 LED器件多应力条件下寿命快速 评估系统模型的 5 个未知参数 $\tau_{\mathrm{r}}, A, B, m$ 和 $n$ 的范 围, 在 $\tau_{\mathrm{r}}, A, B, m$ 和 $n$ 取值区间内随机产生 5 个参数 $\tau_{r}=5.3549 \times 10^{-4} \mathrm{~h} \mathrm{~A}^{\mathrm{n}}, A=1.5583 \times 10^{-6}, B=1.8868 \times 10^{-6}$, $m=0.2598$ 和 $n=0.8200$. 利用这 5 个随机产生的参数和 激活能 $E_{\mathrm{a}}=0.6889 \times 10^{-19} \mathrm{~J}$, 虚构一个 LED器件多应力条 件下寿命快速评估系统模型为

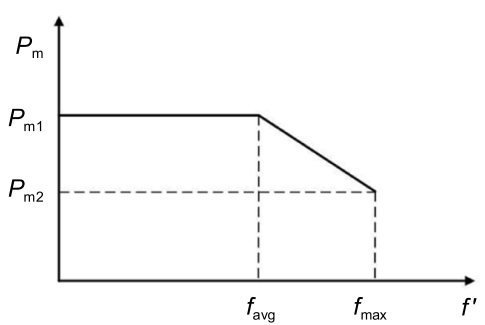

图 4 自适应变异概率 


$$
\begin{aligned}
\tau(I, H, T)= & 5.3549 \times 10^{-4} \mathrm{e}^{\frac{4989.86}{T}} I^{-0.82} H^{-0.2598} \\
& +8.3445 \times 10^{-10} \mathrm{e}^{\frac{9979.72}{T}} I^{-1.64} \\
& +1.0104 \times 10^{-9} \mathrm{e}^{\frac{9979.72}{T}} H^{-0.5196} .
\end{aligned}
$$

迄今, 还未见公开报道的有关LED器件多应力条 件下寿命快速评估系统模型的文献参考, 式(12)虚构 模型的 5 个随机产生参数与实际LED器件的预测寿命 不存在相关性. 为验证提出的自适应遗传算法的有效 性, 对式(12)的虚构模型进行仿真研究. 设计的自适 应遗传算法的种群大小选为 100 , 最大进化代数取为 1000 次, 通过随机方式产生初始种群.

根据式(12)的LED器件多应力条件下寿命快速评 估系统模型的输入量范围, 随机生成 4 组和 20 组的温 度 $T$, 湿度 $H$ 和电流 $I$ 输入数据. 将生成的这 4 组和 20 组 输入数据输入式(12)模型, 即可获得相应的4组和 20 组 预测寿命的输出数据. 使用生成的这 4 组和 20 组输入 输出数据, 构建 4 组和 20 组样本数据. 将温度 $T$, 湿度 $H$ 和电流 $I$ 作为式(12)的输入量, 计算得到的式(12)输出 量定义为虚拟寿命.

假设式(12)模型的 5 个参数 $\tau_{\mathrm{r}}, A, B, m$ 和 $n$ 为未知参 数, 利用构建的 4 组和 20 组样本数据, 基于MATLAB 软 件采用. M语言编写基于自适应遗传算法的LED器件 多应力条件下寿命快速评估系统建模程序, 辨识这 5 个 未知参数. 使用得到的 5 个辨识参数, 建立与式(12)虚 拟模型吻合的LED器件多应力条件下寿命快速评估 系统模型, 应用建立的模型可预测任意温度 $T$ 、湿度 $H$ 和电流 $I$ 状态下的LED寿命.

为了验证基于自适应遗传算法的LED器件多应 力条件下寿命快速评估系统建模的有效性和正确性, 预测中高温中高湿中高电流(温度 $T=330 \mathrm{~K}$, 湿度 $H=50$ $\% \mathrm{RH}$, 电流 $I=0.5 \mathrm{~A}$ ) 状态下的LED寿命 $\tau_{1}$, 分析比较该预 测寿命与虚拟寿命 $\left(8.11 \times 10^{4} \mathrm{~h}\right)$; 再次预测常温常湿常 电流下 (温度 $T=298.15 \mathrm{~K}$, 湿度 $H=22.5 \% \mathrm{RH}$, 电流 $I=0.35$ A) 的LED寿命 $\tau_{2}$, 分析比较常温常湿常电流下的预测 寿命与虚拟寿命 $\left(2.44 \times 10^{7} \mathrm{~h}\right)$. 为了验证所设计的自适 应遗传算法的收玫性, 分别对 4 组和 20 组样本数据下 建模程序运算 10 次.

图 5 为 4 组和 20 组样本数据构建的自适应遗传算 法的种群最优个体适应度与迭代次数之间的关系曲 线, 种群最优个体适应度随着迭代次数的增加而增 大, 进而逐渐减小预测寿命与虚拟寿命之间的误差.
图6 10 分别是 $\tau_{\mathrm{r}}, A, B, m$ 和 $n$ 参数与迭代次数之间的关 系曲线, $\tau_{\mathrm{r}}, A, B, m$ 和 $n$ 参数随着迭代次数增加而不断 趋于真实值.

所设计的自适应遗传算法是一个全局寻优算法, 最终目的是寻找最优的预测寿命与虚拟寿命误差的目

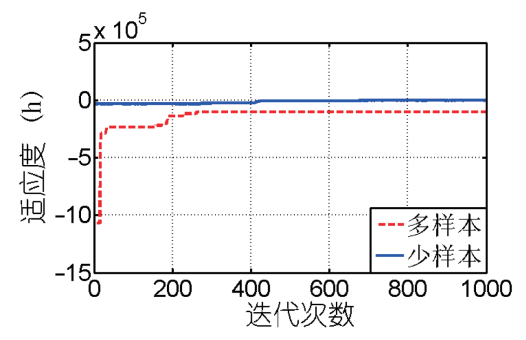

图 5 (网络版彩图)种群最优个体的适应度曲线

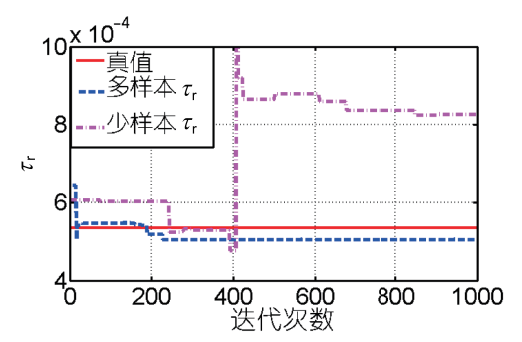

图 6 (网络版彩图) 参数 $\tau_{\mathrm{r}}$ 辨识曲线

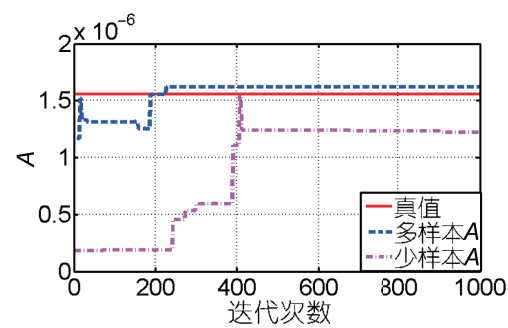

图 7 (网络版彩图) 参数 $A$ 辨识曲线

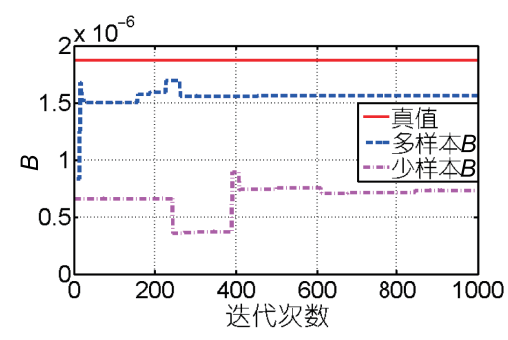

图 8 (网络版彩图)参数 $B$ 辨识曲线 


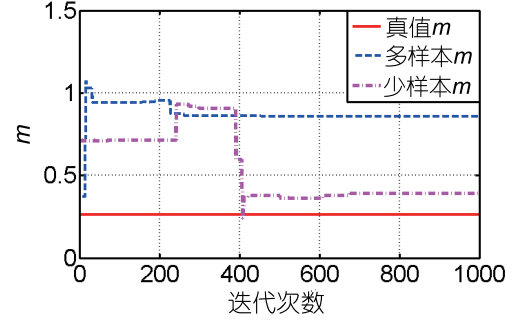

图 9 (网络版彩图)参数 $m$ 辨识曲线

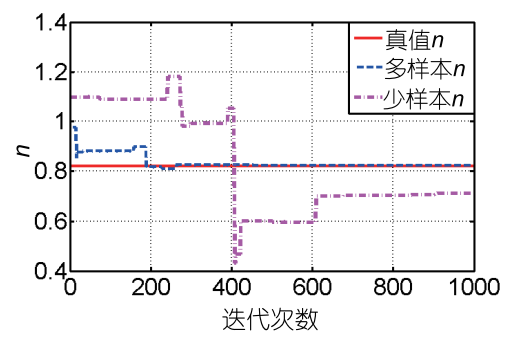

图 10 (网络版彩图)参数 $n$ 辨识曲线

标函数, 在运算过程中寻找使目标函数最优的 5 个辨 识参数 $\tau_{\mathrm{r}}, A, B, m$ 和 $n$, 在达到最优目标函数的同时, 辨 识的 5 个参数只是该算法计算的必然结果. 因此, 相比 于预测寿命与虚拟寿命之间的误差, 辨识参数与真实 参数之间的误差较大.

基于 4 组样本数据构建的LED器件多应力条件下 寿命快速评估系统模型, 中高温中高湿中高电流和常 温常湿常电流下的预测寿命与虚拟寿命之间的相对误 差如表 1 所示. 10 次实验中, $\tau_{\mathrm{r}}, A, B, m$ 和 $n$ 辨识参数的平 均值分别为 $5.7651 \times 10^{-4} \mathrm{~h} \mathrm{~A}{ }^{\mathrm{n}}, 0.6907 \times 10^{-6}, 1.3335 \times 10^{-6}$,
1.5466和 0.8320 . 在中高温中高湿中高电流状态下, 预 测寿命与虚拟寿命之间的相对误差平均值为 $37.8 \%$. 在常温常湿常电流状态下, 预测寿命与虚拟寿命之间 的相对误差平均值为 $2.8 \%$.

基于 20 组样本数据构建的LED器件多应力条件下 寿命快速评估系统模型, 中高温中高湿中高电流和常 温常湿常电流下的预测寿命与虚拟寿命之间的相对误 差如表1所示. 10 次实验中, $\tau_{\mathrm{r}}, A, B, m$ 和 $n$ 辨识参数的平 均值分别为 $3.6495 \times 10^{-4} \mathrm{~h} \mathrm{~A}^{\mathrm{n}}, 1.0451 \times 10^{-6}, 0.9628 \times 10^{-6}$, 1.7009和 1.1716. 在中高温中高湿中高电流状态下, 预 测寿命与虚拟寿命之间的相对误差平均值为 $21.4 \%$. 在常温常湿常电流状态下, 预测寿命与虚拟寿命之间 的相对误差平均值为 $18.3 \%$.

仿真结果表明: 设计的自适应遗传算法具有收敛 性; 相比于基于 4 组样本数据建立的LED器件多应力 条件下寿命快速评估系统模型, 20 组样本数据建立的 模型的辨识参数更接近于真实参数; 在中高温中高湿 中高电流状态下, 相比于基于 4 组样本数据建立的LED 器件多应力条件下寿命快速评估系统模型, 20 组样本 数据建立的模型具有更精确的预测寿命; 在常温常湿 常电流状态下, 相比于基于 20 组样本数据建立的LED 器件多应力条件下寿命快速评估系统模型, 4 组样本 建立的模型具有更精确的预测寿命; 设计的自适应遗 传算法具有可行性和有效性.

\subsection{LED加速寿命试验系统}

为了获得样本数据从而建立LED器件多应力条件 下寿命快速评估系统模型, 需要对LED器件进行加速

\section{表 1 预测寿命与虚拟寿命之间的相对误差}

\begin{tabular}{|c|c|c|c|c|}
\hline & \multicolumn{2}{|c|}{ 4组样本数据 } & \multicolumn{2}{|c|}{20 组样本数据 } \\
\hline & $\tau_{1}$ & $\tau_{2}$ & $\tau_{1}$ & $\tau_{2}$ \\
\hline 仿真1相对误差 & $29.00 \%$ & $5.20 \%$ & $6.10 \%$ & $9.80 \%$ \\
\hline 仿真 2 相对误差 & $36.90 \%$ & $3.70 \%$ & $16.50 \%$ & $8.80 \%$ \\
\hline 仿真3相对误差 & $52.00 \%$ & $1.40 \%$ & $20.50 \%$ & $2.30 \%$ \\
\hline 仿真4相对误差 & $32.20 \%$ & $2.20 \%$ & $25.70 \%$ & $35.30 \%$ \\
\hline 仿真 5 相对误差 & $43.10 \%$ & $4.50 \%$ & $22.10 \%$ & $54.90 \%$ \\
\hline 仿真6相对误差 & $42.30 \%$ & $4.20 \%$ & $25.70 \%$ & $0.90 \%$ \\
\hline 仿真7相对误差 & $37.30 \%$ & $2.60 \%$ & $26.30 \%$ & $9.20 \%$ \\
\hline 仿真 8 相对误差 & $34.70 \%$ & $2.00 \%$ & $25.20 \%$ & $27.60 \%$ \\
\hline 仿真9相对误差 & $30.40 \%$ & $0.70 \%$ & $25.00 \%$ & $31.00 \%$ \\
\hline 仿真 10 相对误差 & $39.80 \%$ & $2.00 \%$ & $20.50 \%$ & $2.70 \%$ \\
\hline 平均相对误差 & $37.80 \%$ & $2.80 \%$ & $21.40 \%$ & $18.30 \%$ \\
\hline
\end{tabular}


寿命试验, 得到不同温度、湿度、电流应力下的LED 寿命.

采用中国晶科、韩国首尔半导体和美国科锐三 个公司的 $1 \mathrm{~W}$ 大功率LED作为LED加速寿命试验的实 验样品, 该实验样品如图 11 所示. 每组 60 个LED均为 同一厂家同一批次样品, 保证初始结构基本一致. 实 验场地为深圳计量质量检测研究院环境实验室.

图12为LED加速寿命试验系统示意图. 系统由高 低温试验箱、LED夹具、系统电源、恒流调节电路 箱、精密电流采样电阻箱和电流参数记录仪组成. 由 于 60 路LED采用并联方式, 在每路LED中加入电流调 节电路, 使每路LED电流值保持在工作电流状态, 则 使每一路LED相互独立, 互不影响. 每路LED的电流 值可以实现实时、准确的监测记录. 为了避免受温度 的影响, 增加整个系统的可靠性, 试验时将LED样品 放入高低温箱进行应力加载作用, 系统其他模块安置 于试验箱外.

LED环境试验平台如图13所示. 采用重庆银河试 验仪器有限公司EBS-SDJ605F型号的高低温试验箱, 该试验箱内尺寸为 $870 \mathrm{~mm}($ 长 $) \times 800 \mathrm{~mm}$ (宽) $\times 700 \mathrm{~mm}$

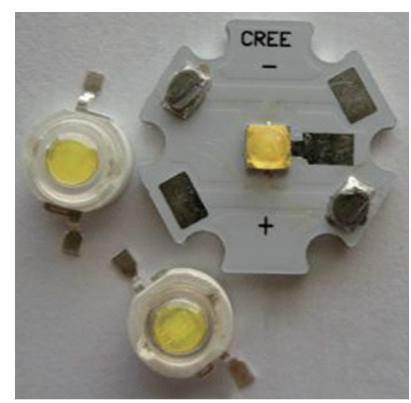

图 11 (网络版彩图)LED器件实验样品

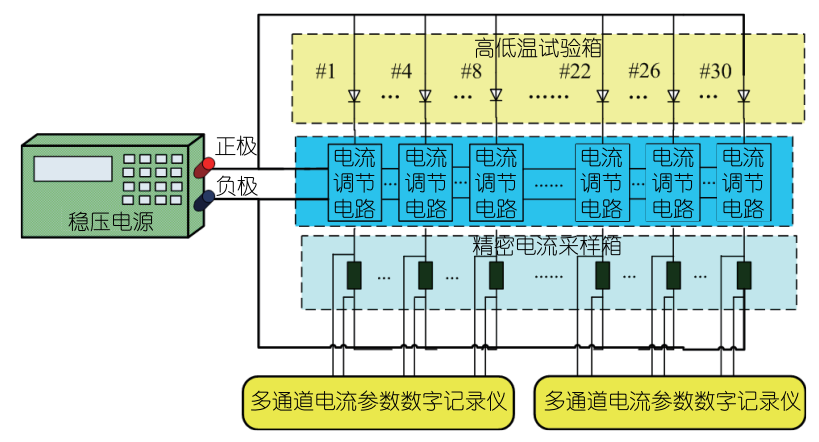

图 12 (网络版彩图)加速寿命试验系统示意图
(高), 模拟温度、湿度范围分别为 $-70 \sim 150^{\circ} \mathrm{C} 、 30 \sim 98$ $\% \mathrm{RH}$, 温度均匀度小于 $2^{\circ} \mathrm{C}$, 温度偏差为 $\pm 2^{\circ} \mathrm{C}$, 湿度偏 差为 $-3 \sim+2 \% \mathrm{RH}$. 使用图 14 的 LED光色电参数测量 系统, 该系统主要包括德国 Instrument Systems公司的 Spectro320光谱仪和ISP 500-211积分球.

参考IESNA的LM-80标准以及某知名厂家的企业 标准, 应力测试设定的实验条件为LED驱动电流为各 路独立的恒定电流驱动, 环境温度和湿度可控.

\section{3 模型的试验验证}

对中国晶科公司的 60 颗 $1 \mathrm{~W}$ 大功率LED样品进行 加速寿命试验, 该 LED样品的激活能为 $E_{\mathrm{a}}=0.6889 \times 10^{-19}$ J. 通过加速寿命试验分别测量温度 $T=338.15 \mathrm{~K}$, 湿度

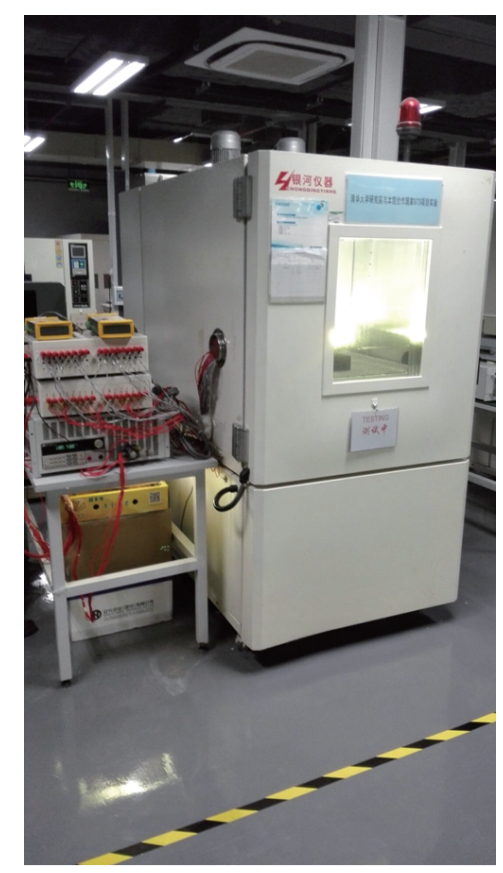

图 13 (网络版彩图)LED环境试验平台

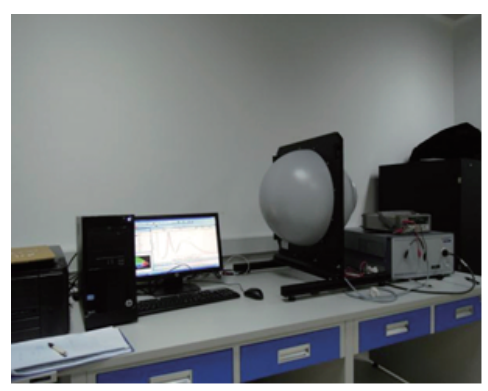

图 14 (网络版彩图)LED光色电参数测量系统 
表 2 预测寿命、预测寿命与实验寿命之间的相对误差

\begin{tabular}{cccccc}
\hline$I(\mathrm{~A})$ & $H(\% \mathrm{RH})$ & $T(\mathrm{~K})$ & 实验寿命 $(\mathrm{h})$ & 预测寿命 $(\mathrm{h})$ & 相对误差 \\
\hline 0.7 & 85 & 338.15 & 1587 & 2497 & $57.35 \%$ \\
0.5 & 85 & 338.15 & 4686 & 3843 & $17.98 \%$ \\
0.35 & 85 & 338.15 & 8141 & 8139 & $0.02 \%$ \\
\hline
\end{tabular}

$H=85 \% \mathrm{RH}$, 电流 $I=0.7 \mathrm{~A}$; 温度 $T=338.15 \mathrm{~K}$, 湿度 $H=85$ $\% \mathrm{RH}$, 电流 $I=0.55 \mathrm{~A}$; 温度 $T=338.15 \mathrm{~K}$, 湿度 $H=85 \% \mathrm{RH}$, 电流 $I=0.35$ A 状态下的LED使用寿命, 将此寿命定义 为LED实验寿命. 获得的LED实验寿命如表 2 所示.

利用表 2 的 3 组实验样本数据, 基于MATLAB软 件采用.M语言编写 LED器件多应力条件下寿命快 速评估系统建模程序, 辨识得到 5 个未知参数 $\tau_{\mathrm{r}}, A$, $B, m$ 和 $n$ 分别为 $\tau_{\mathrm{r}}=4.97878 \times 10^{-4} \mathrm{~h} \mathrm{~A}^{\mathrm{n}}, A=-0.91965 \times 10^{-8}$, $B=-1.0796 \times 10^{-8}, m=0.39485$ 和 $n=1.8729$, 建立LED器件 多应力条件下寿命快速评估系统模型为

$$
\begin{aligned}
\tau(T, H, I)= & 4.97878 \times 10^{-4} \mathrm{e}^{\frac{4989.6831113}{T}} I^{-1.8729} H^{-0.39485} \\
& -4.57875 \times 10^{-12} \mathrm{e}^{\frac{9979.366226}{T}} I^{-3.7458} \\
& -5.37492 \times 10^{-12} \mathrm{e}^{\frac{9979.366226}{T}} H^{-0.7897}
\end{aligned}
$$

采用式(13)的LED器件多应力条件下寿命快速评 估系统模型, 计算得到的温度 $T=338.15 \mathrm{~K}$, 湿度 $H=85$ $\% \mathrm{RH}$, 电流 $I=0.7 \mathrm{~A}$; 温度 $T=338.15 \mathrm{~K}$, 湿度 $H=85 \% \mathrm{RH}$, 电流 $I=0.55 \mathrm{~A}$; 温度 $T=338.15 \mathrm{~K}$, 湿度 $H=85 \% \mathrm{RH}$, 电流 $I=0.35 \mathrm{~A}$ 状态下的预测寿命、预测寿命与实验寿命之 间的相对误差如表2所示, 预测寿命与实验寿命之间 的相对误差分别为 $57.35 \%, 17.98 \%$ 和 $0.02 \%$. 计算得到 的常温常湿常电流状态下的预测寿命为 $32515 \mathrm{~h}$, 接近 于LED行业平均技术水平的预期寿命值 $30000 \mathrm{~h}$. 研究 结果表明建立的LED器件多应力条件下寿命快速评 估系统模型具有有效性.

LED器件多应力条件下寿命快速评估系统模型是 基于样本数据的建模, 因而其预测寿命的准确性严重 依赖于样本数据的准确性和个数. 因此, 利用加速寿 命试验得到的 3 组样本数据, 获得的表 2 所示的预测寿 命与实验寿命之间的相对误差较大. 通过增大试验样 本数据的个数、提高试验样本数据在温度、湿度和 电流状态空间的覆盖率, 可提高LED器件多应力条件 下寿命快速评估系统模型的准确性和适用性.

采用图15所示的国家重点基础研究发展计划(973 计划)成果(LED器件多参数复合过应力测试系统)进行

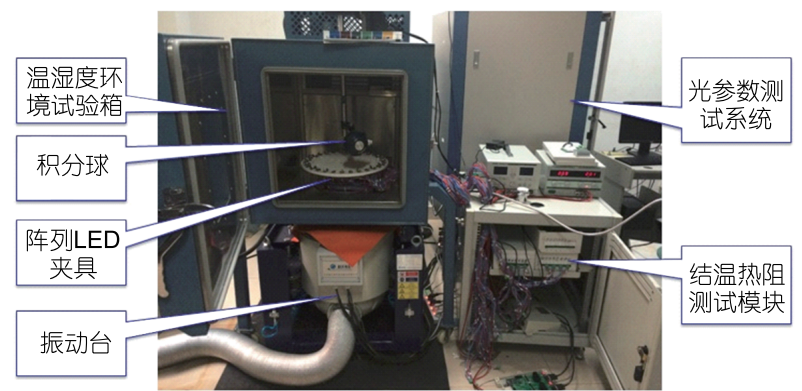

图 15 (网络版彩图)LED器件多参数复合过应力测试系统

实验验证. 该系统可作为提出的模型的配套设备, 使 企业针对每种 LED器件产品均能在线、快速、较准 确地实现寿命测评.

\section{5 结论}

本文以Arrhenius单应力环境因素模型和Peck模型 为基础, 提出了一种LED器件多应力条件下寿命快速 评估系统模型. 该模型采用设计的自适应遗传算法辨 识模型的未知参数, 以预测任意环境温度、湿度和驱 动电流应力下的LED寿命, 特别是常温常湿常电流应 力下的LED寿命. 对设计的自适应遗传算法进行了仿 真, 得到了合理的虚构LED器件寿命快速评估系统模 型参数, 获得的LED预测寿命接近于LED虚拟寿命, 验 证了设计的自适应遗传算法的有效性. 采用加速寿命 试验样本数据构建了 LED器件多应力条件下寿命快 速评估系统模型, 采用该模型计算得到的预测寿命与 实验寿命之间的相对误差较小, 计算得到的常温常湿 常电流应力下的LED器件预测寿命为 $32515 \mathrm{~h}$, 接近于 LED行业平均技术水平的 $30000 \mathrm{~h}$ 预期寿命. LED器件 多应力条件下寿命快速评估系统模型可较准确地预 测LED器件寿命, 并可继续拓展成为纳入更多应力(如 振动、温度冲击等)的模型. 使用提出的模型并结合 所开发的加速寿命试验设备, 企业可以快速较准确地 实现对LED器件的寿命测评. 


\section{参考文献}

1 Shagerdmootaab A, Moallem M. A double-loop primary-side control structure for HB-LED power regulation. IEEE Trans Power Electron, 2016, 31: $2476-2484$

2 Qiu Y J, Wang L L, Wang H L, et al. Bipolar ripple cancellation method to achieve single-stage electrolytic-capacitor-less high-power LED driver. IEEE J Emerg Sel Topics Power Electron, 2015, 3: 698-713

3 Pollock A, Pollock H, Pollock C. High efficiency LED power supply. IEEE J Emerg Sel Topics Power Electron, 2015, 3: 617-623

4 Lee D W, Cho S W, Kim Y J. Numerical study on the heat dissipation characteristics of high-power LED module. Sci China Technol Sci, 2013, 56: $2150-2155$

5 王军喜, 间建昌, 郭亚楠, 等. 氮化物深紫外LED研究新进展. 中国科学: 物理学 力学 天文学, 2015, 45: 067303

6 Chen H T, Tan S C, Hui S Y R. Nonlinear dimming and correlated color temperature control of bicolor white LED systems. IEEE Trans Power Electron, 2015, 30: 6934-6947

7 Lin R L, Tsai J Y, Liu S Y, et al. Optimal design of LED array combinations for CCM single-loop control LED drivers. IEEE J Emerg Sel Topics Power Electron, 2015, 3: 609-616

8 Hui S Y, Li S N, Tao X H, et al. A novel passive offline LED driver with long lifetime. IEEE Trans Power Electron, 2010, 25: 2665-2672

9 和倩, 赵敏, 徐立生. LED应用产品的平均寿命评估方法. 加工、测量与设备, 2013, 50: 466-469

10 赵阿玲, 尚守锦, 陈建新. 大功率白光LED寿命试验及失效分析. 照明工程学报, 2010, 21: 48-57

11 陈宇, 黄帆, 严华锋, 等. 通过加速老化实验对LED器件可靠性的研究. 照明工程学报, 2011, 22: 34-38

12 郭伟玲, 訤星, 崔德胜, 等. 一种基于伪失效寿命的LED可靠性快速评价方法. 发光学报, 2013, 34: 213-217

13 Shei S C, Sheu J K, Shen C F. Improved reliability and ESD characteristics of flip-chip GaN-based LEDs with internal inverse-parallel protection diodes. IEEE Electron Device Lett, 2007, 28: 346-349

14 么东阁, 陈建新, 间静. 大功率白光LED的应用及其可靠性研究. 照明工程学报, 2011, 22: 43-46

15 黄伟明, 文尚胜, 夏云云. 基于BP神经网络的LED可靠性模型研究. 发光学报, 2015, 36: 962-968

16 Jenkins D, Bhargava A. LED Lighting: Maximizing the reliability, safety, and efficiency of light fixtures in hazardous environments. IEEE Ind Appl Mag, 2015, 21: 64-67

17 Suhir E. Accelerated life testing in photonics packaging: Its objectives, role, attributes, challenges, pitfalls, predictive models, and interaction with other accelerated stress categories. Proc SPIE Brugge, 2003, 4947: 175-196

18 贺卫利, 郭伟玲, 高伟, 等. 大功率发光二极管可靠性和寿命评价试验方法. 应用光学, 2008, 29: 533-536

19 余菲, 金雷. GaN发光二极管的老化数学模型及寿命测试方法. 中国激光, 2011, 38: 0806001

20 IES. IES LM-80-08: Approved method: Measuring lumen maintenance of LED light sources, 2008

21 IES. IES TM-21-11: Projecting long term lumen maintenance of LED packages, 2011

22 Srinivas M, Patnaik L M. Adaptive probabilities of crossover and mutation in genetic algorithms. IEEE Trans Syst Man Cybern, 1994, 24: 656-667

23 Wang F, Li J, Liu S, et al. An improved adaptive genetic algorithm for image segmentation and vision alignment used in microelectronic bonding. IEEE/ASME Trans Mechatron, 2014, 19: 916-923

24 Du Y, Fang J, Miao C. Frequency-domain system identification of an unmanned helicopter based on an adaptive genetic algorithm. IEEE Trans Ind Electron, 2014, 61: 870-881

25 Wei X K, Zhang C, Wang B Z, et al. Improved self-adaptive genetic algorithm with quantum scheme for electromagnetic optimisation. IET Microw Antenn P, 2014, 8: 965-972

26 Huang S D, Cao G Z, He Z Y, et al. Maximum-force-per-ampere strategy of current distribution for efficiency improvement in planar switched reluctance motors. IEEE Trans Ind Electron, 2016, 63: 1665-1675 


\title{
Modeling of the rapid lifetime assessment system with multiple stresses for LED devices using adaptive genetic algorithm
}

\author{
HUANG SuDan ${ }^{1,2}$, LIU HuaiYuan ${ }^{1}$, CAO GuangZhong ${ }^{2}$, ZHOU Lin ${ }^{1}$, WU HaiLin ${ }^{3}$, HU YiMin ${ }^{1}$, \\ JING Gang ${ }^{1}$, CAO MingGao ${ }^{1}$, XIAO WenPeng ${ }^{1} \&$ LIU Yan $^{1}$ \\ ${ }^{1}$ Guangdong Optomechatronics Key Laboratory, Shenzhen Optomechatronics Key Laboratory, Research Institute of Tsinghua University in Shenzhen, \\ Shenzhen 518060, China; \\ ${ }^{2}$ Shenzhen Key Laboratory of Electromagnetic Control, Shenzhen University, Shenzhen 518060, China; \\ ${ }^{3}$ College of Materials Science and Engineering, Shenzhen University, Shenzhen 518060, China
}

The light-emitting diode (LED) is a promising candidate for the lighting, instrument reading, etc., due to its features of high efficiency, long lifetime, small size, fast response, anti vibration, low-voltage operation, and environmental protection, which is an emerging cold light source. This paper proposes a novel model of the rapid lifetime assessment system with multiple stresses for LED devices, to solve the problems of the complex environmental stresses imposed on the lifetime of LED devices, the model of accelerated life testing without consideration of multiple stresses, and the low-precision lifetime assessment model. This model is suitable for all types of LED devices. Taking the operating time to $30 \%$ of lumen depreciation as the lifetime of LED devices, the environmental temperature and humidity, and input current are the inputs of this model, and the lifetime of the LED device is the output of this model. The parameters of this model are determinated by using the designed adaptive genetic algorithm. A several number of sample data is first obtained through accelerated life testing under the condition of high temperature, humidity, and current. The parameters of this model are then identified with the obtained sample data. This model is further built by applying the identified parameters. Additionally, the lifetime evaluation is carried out via this model under the condition of normal temperature, humidity, and current. Research results demonstrate that the proposed model can evaluate the lifetime of LED devices fast with a comparatively high accuracy; the evaluated lifetime of the proposed model is close to the realizable life expectancy of the LED industry under the condition of normal temperature, humidity, and current; the practicability of the proposed model for lifetime reliability evaluation of LED devices is verified; the proposed model can be further developed with much more stresses.

light-emitting diode, rapid lifetime assessment, accelerated life testing, adaptive genetic algorithm, parameter identification

doi: $10.1360 / \mathrm{N} 092016-00058$ 